\title{
Higher Order Statistics and Phase Synchronization as Features in a Motor Imagery Paradigm
}

\author{
Oana-Diana Hrisca-Eva ${ }^{1}$, Anca Mihela Lazar ${ }^{3}$ \\ Faculty of Medical Bioengineering \\ “Grigore T. Popa” University of Medicine and Pharmacy \\ Iasi, Romania
}

\author{
Madalina-Giorgiana Murariu ${ }^{2}$ \\ Faculty of Electronics, Telecommunications and Information \\ Technology, "Gheorghe Asachi” Technical University \\ Iasi, Romania
}

\begin{abstract}
The paper proposes an approach based on higher order statistics and phase synchronization for detection and classification of relevant features in electroencephalographic (EEG) signals recorded during the subjects are performing motor tasks. The method was tested on two different datasets and the performance was evaluated using $k$ nearest neighbor classifier. The results (classification rates higher than $90 \%$ ) have shown that the method can be used for discriminating right and left motor imagery tasks as an offline analysis for EEG in a brain computer interface system.
\end{abstract}

Keywords-Brain computer interface; motor imagery; higher order statistics; phase synchronization; EEG

\section{INTRODUCTION}

The brain computer interface (BCI) system has aroused a real interest, as it has become an important tool in translating the measured brain activity into control commands.

The BCI was developed for biomedical applications, which led to the development of assistive devices for restoring movement and communication force for patients with disabilities. The use of electroencephalography (EEG) in the state-of-the-art of brain-computer interface technology has expanded to enhance quality of life, with medical and nonmedical applications [1]. Various BCI innovations, such as the BCI wheelchair [2], spellings, BCI exoskeleton [3], BCI prosthesis, BCI robot [4] and others are the result of this huge interest in BCI [5].

The electroencephalogram is a source of information often used in BCI because it records the electrical activity of the brain with the help of the attached electrodes on the scalp. The BCI system amplifies the collected signals, notifies these changes and transforms them into control signals for communication or control of external equipment. BCI allows patients with paralysis or motor disorders to have an alternative method of communication and control.

Compared with other existing neuroimaging modalities, electroencephalogram has higher time resolution, portability, availability, and, so it is more commonly used.

EEG based BCI systems are centered on several paradigms [6-7]: Steady State Visual Evoked Potential (SSVEP), P300, Slow Cortical Potential (SCP) and Motor Imagery (MI). Motor imagery implies imagination of hand/arm/finger/foot movement. More precisely, motor imagery is the translation of the motor intention into control signals for a BCI device. After imagination, event-related synchronization (ERS) and desynchronization (ERD) are produced over the sensorimotor cortex region [8]. Brain activity recorded via EEG is classified into five different rhythms: Delta $(0-4 \mathrm{~Hz})$, Theta $(4-7 \mathrm{~Hz}), \mathrm{Mu}$ (8-12 Hz), Beta (12-30 Hz), Gamma (30-70 Hz). Mu and Beta rhythms are associated with actions like preparation and movement of limbs.

Methods like autoregressive model [9], independent component analysis [10], wavelet transform [11] have been applied in order to extract relevant features contained in the EEG signal while a motor imagery task was performed.

The aim of the paper is to propose a combination of features which includes higher-order statistics (HOS) based on bispectrum and bicoherence and phase synchronization based on phase locking value and phase lag index. The features can be used for discriminating motor tasks in a motor imagery paradigm.

In Section II the handled datasets are described and Section III presents the methods, features extraction and classification. Section IV belongs to results and Section V to conclusions.

\section{DATASETS DESCRIPTION}

Dataset I contains EEG signals acquired in our laboratory. The brain activity was recorded using a gMobilab+ module [12] and BCI 2000 platform [13]. Dataset II was downloaded from the internet [14]. Table I presents the information regarding the manipulated datasets.

TABLE I. DATABASES SUMMARY

\begin{tabular}{|l|l|l|}
\hline Database information & Dataset I & Dataset II \\
\hline Number of subjects & 57 & 9 \\
\hline Channels & $\mathrm{C} 3, \mathrm{C} 4, \mathrm{CP} 3, \mathrm{CP} 4, \mathrm{P} 3, \mathrm{P} 4, \mathrm{Cz}$ \\
\hline Number of Trials & 60 & 90 \\
\hline Sampling frequency & $256 \mathrm{~Hz}$ & $100 \mathrm{~Hz}$ \\
\hline Paradigm description & $\begin{array}{l}\text { On a monitor screen right and left arrows were } \\
\text { displayed. } \\
\text { The subjects had to perform the motor task } \\
\text { indicated by the arrow (left hand and right hand } \\
\text { motor imagery). }\end{array}$ \\
\hline
\end{tabular}




\section{METHODS}

Higher order spectra are expressed in terminologies of higher order statistics such as moments and cumulants of a random process, in this case EEG signals [15-16].

Bispectrum is commonly applied in the biomedical area mainly for EEG signal processing. Bispectrum is $\mathrm{d}$ in the bifrequency domain [15]:

$B\left(f_{1}, f_{2}\right)=E\left[X\left(f_{1}\right) X\left(f_{1}\right) X^{*}\left(f_{1}+f_{2}\right)\right]$

where $X(f)$ is the Fourier transform of a windowed portion of a single realization of the EEG signal $x(n T), n$ is an integer index, $T$ is the sampling period and $E[$.$] represents the$ expectation operation.

Bicoherence is given by the following relationship [15-16]:

$B_{\text {norm }}\left(f_{1}, f_{2}\right)=\frac{E\left[X\left(f_{1}\right) X\left(f_{1}\right) X^{*}\left(f_{1}+f_{2}\right)\right]}{\sqrt{P\left(f_{1}\right) P\left(f_{2}\right) P\left(f_{1}+f_{2}\right)}}$

where $P(f)$ is the power spectrum of $x(n T)$.

When the oscillatory phases from two brain regions are correlated the phase synchronization appears. The phase synchronization from the motor imagery period is different from the phase synchronization in the relaxation period so it can be exploited by BCI applications [17].

Large scale synchronization appears between brain signals acquired from electrodes located in the primary motor area and from electrodes located in the additional motor area.

Phase locking value (PLV) [17] and phase lag index (PLI) [18] are calculated in order to detect changes in the large scale synchronization and in order to measure the synchronization between two signals $x(t)$ and $y(t)$.

PLV represents the lastingness of the phase difference between instantaneous phases $\varphi_{x}(t)$ and $\varphi_{y}(t)$ :

$P L V=\left|\left\langle e^{j \Delta \varphi(t)}\right\rangle\right|$

$\Delta \varphi(t)=\varphi_{y}(t)-\varphi_{x}(t)$

The phase lag index is defined by:

$P L I=\left|\left\langle\operatorname{sign}\left[\Delta \theta\left(t_{k}\right)\right]\right\rangle\right|$

sign is the signum function and $<.>$ denotes the time average.

\section{A. Data Processing for Higher Order Statistics}

Two sets of data were formed: one related to right hand movement imagery and the other corresponding to left hand movement imagery.

First, the EEG signals aquired from channels C3, C4, CP3, $\mathrm{CP} 4, \mathrm{P} 3, \mathrm{P} 4$ when the subject imagined the right hand movement were segmented in 30 (Dataset I) and 45 (Dataset II). The bispectrum and bicoherence were estimated using functions from HOSA toolbox [19]. Two different frequency ranges were extracted for analysis in the bi-frequency plane (f1 - f2): $\mathrm{Mu}-\mathrm{Mu}$, Beta - Beta. The module of the bispectrum was calculated for these frequency ranges. In the next step only the elements above the main diagonal (those below the main diagonal being equal to those above) were extracted for the two frequency ranges.

A direct method is applied to estimate the bicoherence.

Four quantity measures for each frequency range were settled: the sum of the bispectrum modules, the sum of the squares bispectrum modules, the sum of the bicoherence modules, the sum of the squares bicoherence modules.

The steps described above were followed for the EEG signals corresponding to left hand motor imagery.

\section{B. Data Processing for Phase Synchronization}

Four sets of data were created: the right hand movement imagery, the rest after right hand movement imagery, the left hand movement imagery and rest after left hand movement imagery. In order to compute PLV and PLI, the instanteneous phase of the EEG signals must be determined. The instanteneous phase of the EEG signals is computed using Hilbert transform. The Hilbert transforms were calculated for the EEG channels mentioned.

One electrode from the auxiliary motor imagery area, $\mathrm{Cz}$, three electrodes from the right hemisphere, $\mathrm{C} 4, \mathrm{CP} 4, \mathrm{P} 4$ and three electrodes from the left hemisphere, C3, CP3, P3 were utilized in order to establish the combinations. The completed pairs of electrodes were: $\mathrm{Cz}-\mathrm{C} 4, \mathrm{Cz}-\mathrm{CP} 4, \mathrm{Cz}-\mathrm{P} 4, \mathrm{Cz}-\mathrm{C} 3, \mathrm{Cz}-$ CP3, Cz-P3. The differences between PLVs of the rest period and motor imagery period were formed for the above pairs of electrodes.

The steps mentioned above were repeated for PLI.

\section{Feature Vectors}

Nine feature vectors were created by bringing together two, three or four of the already mentioned measures. So, the first feature vector is formed by bicoherence and PLV, the second by bicoherence and PLI, the third by bicoherence, PLV and PLI, the fourth by bispectrum and PLV, the fifth by bispectrum and PLI, the sixth by bispectrum, PLV and PLI, the seventh by bicoherence, bispectrum and PLV, the eighth by bicoherence, bispectrum and PLI and the ninth by bicoherence, bispectrum, PLV and PLI. These nine feature vectors were named according to their way of grouping: Bicoherence_PLV was attributed for the feature vector combining bicoherence and PLV, Bicoherence_PLI for the feature vector merging bicoherence and PLI, and so on.

The discrimination between right and left motor imagery was evaluated with $\mathrm{k}$ nearest neighbor ( $\mathrm{kNN}$ ) [20]. The performance of classifier was assessed through classification rate, sensitivity and specificity. The classification rate, the specificity and the sensitivity are characterized using the following terms: true positive (TP), true negative (TN), false negative $(\mathrm{FN})$ and false positive (FP) [21]:

$$
\begin{aligned}
& \text { Classification rate }=\frac{T N+T P}{T N+T P+F N+F P} \\
& \text { Sensitivity }=\frac{T P}{T P+F N} \\
& \text { Specificity }=\frac{T N}{T N+F P}
\end{aligned}
$$




\section{RESULTS}

In Fig. 1 is displayed the bispectrum of an EEG signal corresponding to right hand motor imagery for channel C3 (Dataset I) and Fig. 2 illustrates the bispectrum of an EEG signal corresponding to left hand motor imagery for channel C4 (Dataset I). The bicoherence of an EEG signal corresponding to right hand motor imagery for channel $\mathrm{C} 3$, respectively the bicoherence of an EEG signal corresponding to left hand motor imagery for channel $\mathrm{C} 4$ are shown in Fig. 3 and Fig. 4.

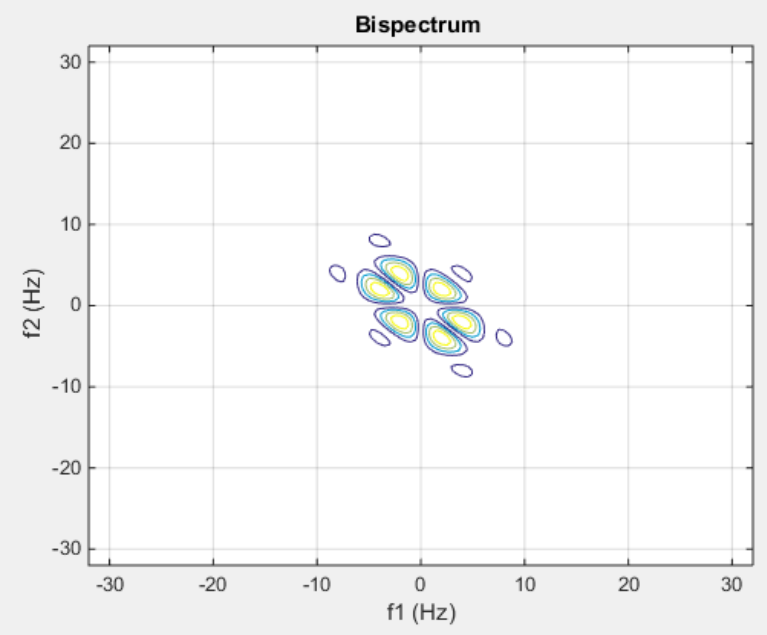

Fig. 1. The Bispectrum of an EEG Signal Corresponding to Right Hand Movement Imagery for Channel C3 - Datatset I.

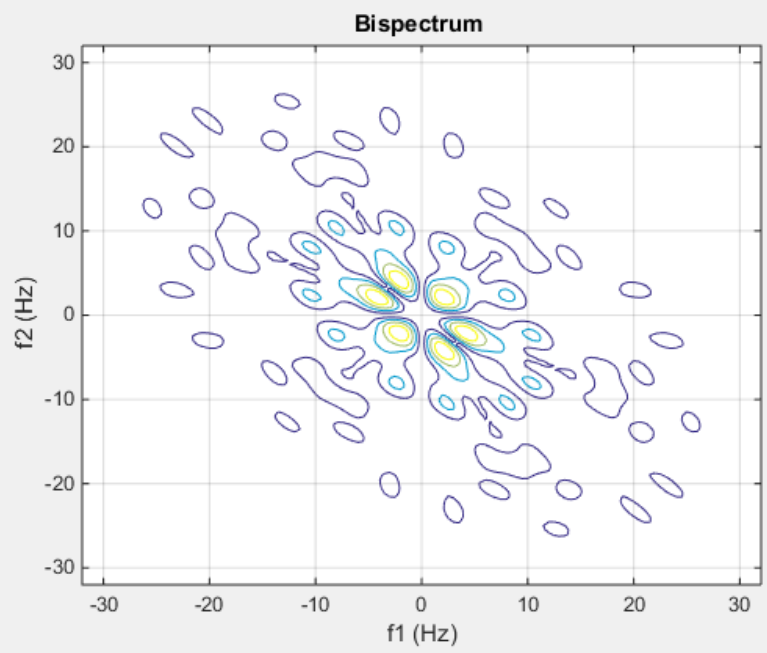

Fig. 2. The Bispectrum of an EEG Signal Corresponding to Left Hand Movement Imagery for Channel C4 - Datatset I.

From the above figures we can see that the bispectrum and the bicoherence yielded to different graphs for EEG signal corresponding to right versus left hand motor imagery and this suggests that can be used as feature vectors for classification.

Fig. 5 shows the box plots for the classification rate, the sensitivity and the specificity using as feature vectors Bicoherence_PLV, Bicoherence_PLI and Bicoherence_PLV_PLI for Dataset I. The highest value (90\%) of the median for classification rates was obtained for
Bicoherence_PLV_PLI and all the subjects attained classification rates in the range $84 \%$ to $94 \%$.

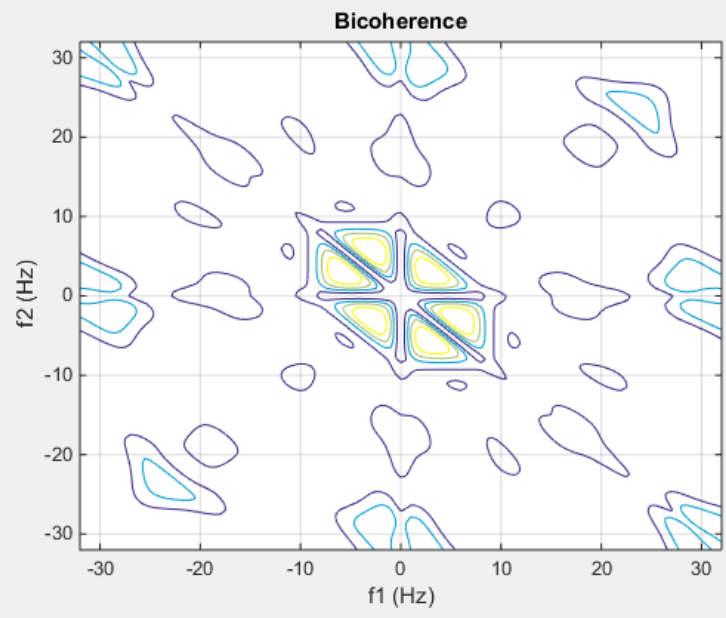

Fig. 3. The Bicoherence of an EEG signal Corresponding to Right Hand Movement Imagery for Channel C3 - Datatset I.

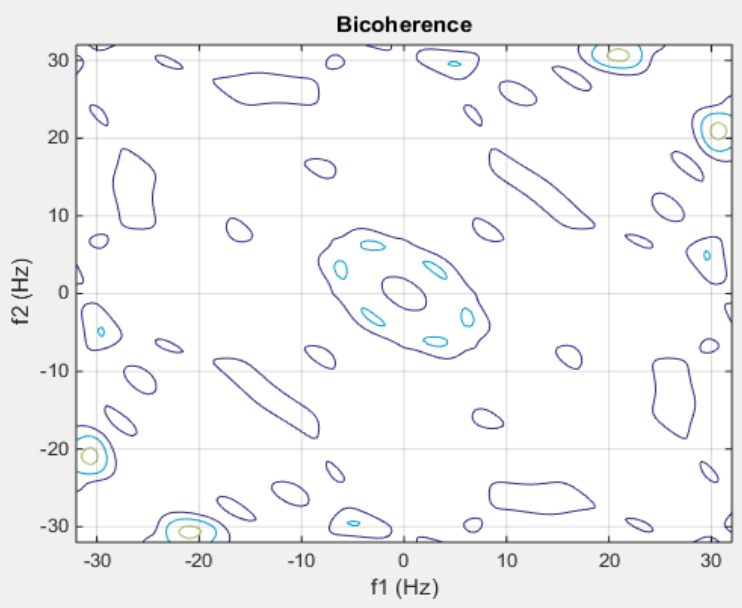

Fig. 4. The Bicoherence of an EEG Signal Corresponding to Left Hand Movement Imagery for Channel C4 - Datatset I.

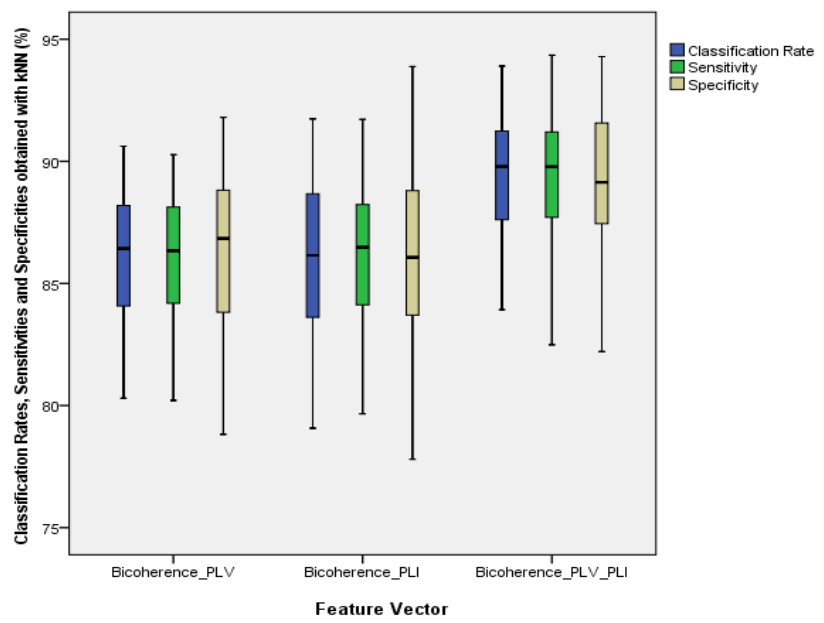

Fig. 5. The Box Plots for Classification Rate, Sensitivity and Specificity using Feature Vector Bicoherence_PLV, Bicoherence_PLI and Bicoherence_PLV_PLI - Dataset I. 
The box plots for the classification rate, the sensitivity and the specificity using as feature vectors Bispectrum_PLV, Bispectrum_PLI and Bispectrum_PLV_PLI - Dataset I are displayed in Fig. 6. The medians for the classification rates, the sensitivities and the specificities obtained were above $88 \%$ for Bispectrum_PLV_PLI. The outliner marked in blue color represents the highest classification rate attained (the value isn't relevant since belongs to a single subject).

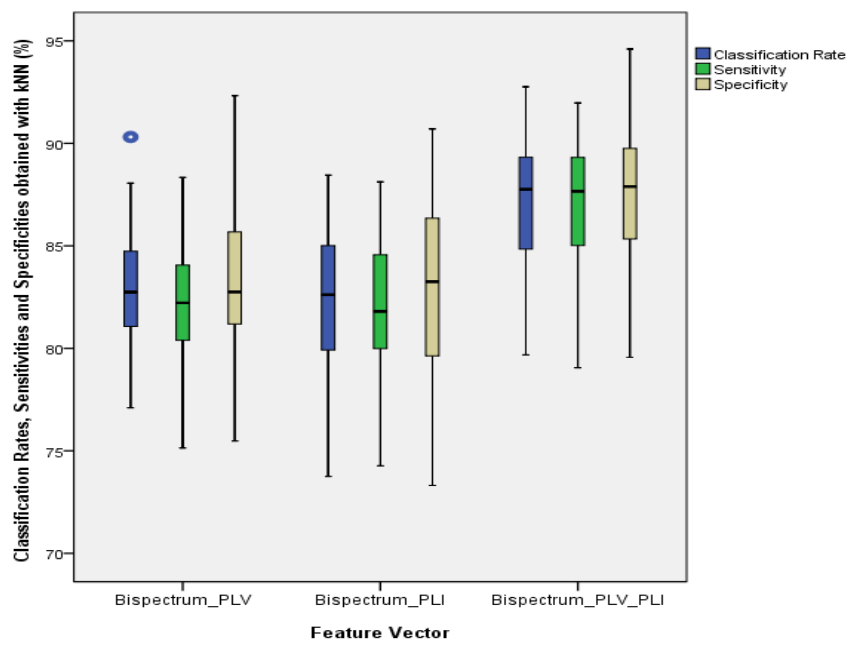

Fig. 6. The Box Plots for Classification Rate, Sensitivity and Specificity using Feature Vector Bispectrum_PLV, Bispectrum_PLI and Bispectrum_PLV_PLI - Dataset I.

In Fig. 7 are presented the box plots for the classification rate, the sensitivity and the specificity using as feature vectors Bicoherence_Bispectrum_PLV, Bicoherence_Bispectrum_PLI and Bicoherence_Bispectrum_PLV_PLI - Dataset I. The highest classification rates, sensitivities and specificities were realized for Bicoherence_Bispectrum_PLV_PLI.

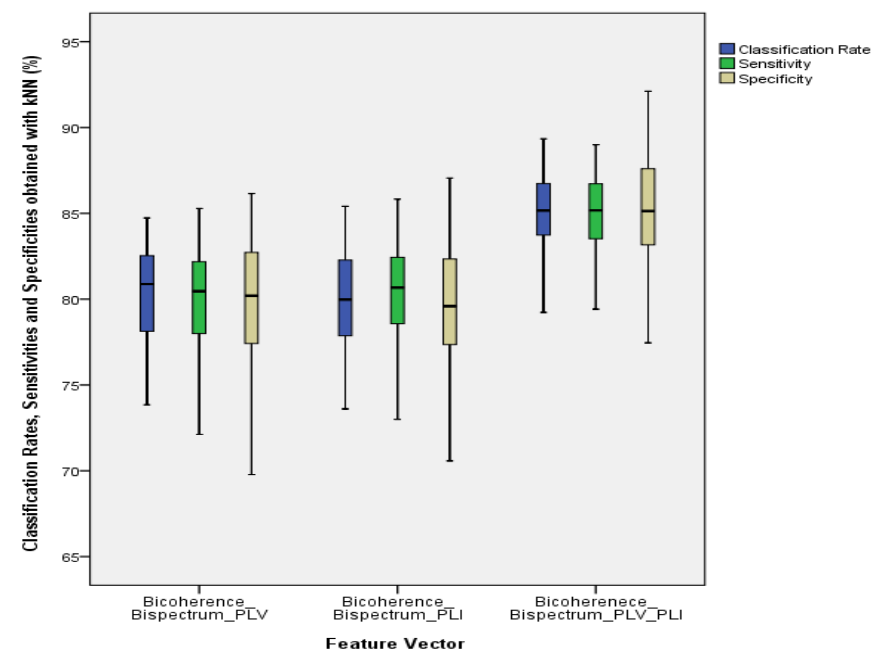

Fig. 7. The Box Plots for Classification Rate, Sensitivity and Specificity using Feature Vector Bicoherence_Bispectrum_PLV,

Bicoherence_Bispectrum_PLI and Bicoherence_Bispectrum_PLV_PLI Dataset I.

Fig. 8 shows the box plots obtained for the classification rate, the sensitivity and the specificity using as feature vector
Bicoherence_PLV,

Bicoherence_PLI

and

Bicoherence_PLV_PLI - Dataset II. The highest classification rates were for Bicoherence_PLV_PLI. The outliners marked with "*” for all the figures are for Subject 9.

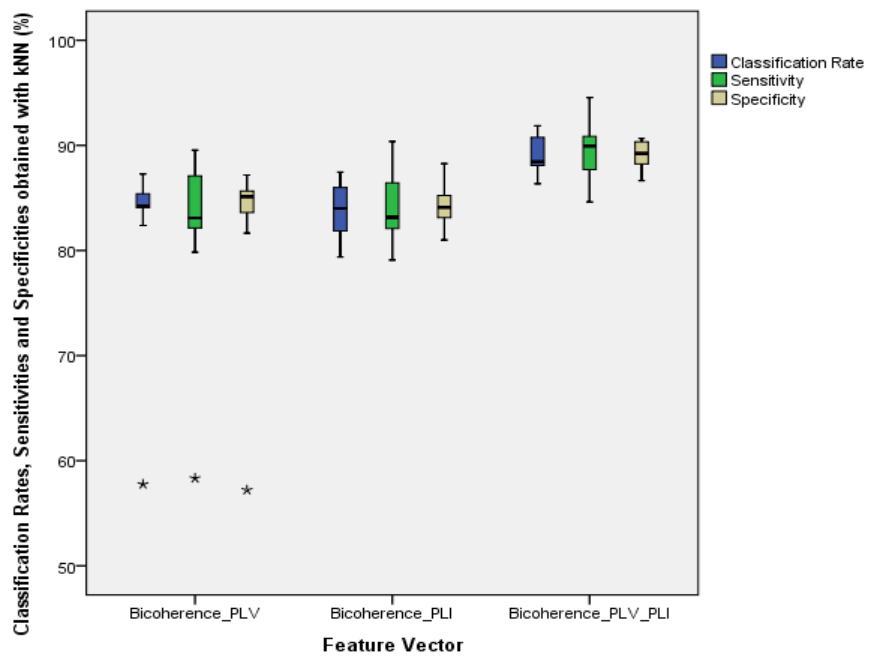

Fig. 8. The Box Plots for Classification Rate, Sensitivity and Specificity using Feature Vector Bicoherence_PLV, Bicoherence_PLI and Bicoherence_PLV_PLI - Dataset II.

Fig. 9 displays the box plots achieved for the classification rate, the sensitivity and the specificity using feature vector Bispectrum_PLV, Bispectrum_PLI and Bispectrum_PLV_PLI - Dataset II. Overall, the best classification rates $(8 \overline{5} \%)$, sensitivities and specificities were obtained for Bispectrum_PLV_PLI .

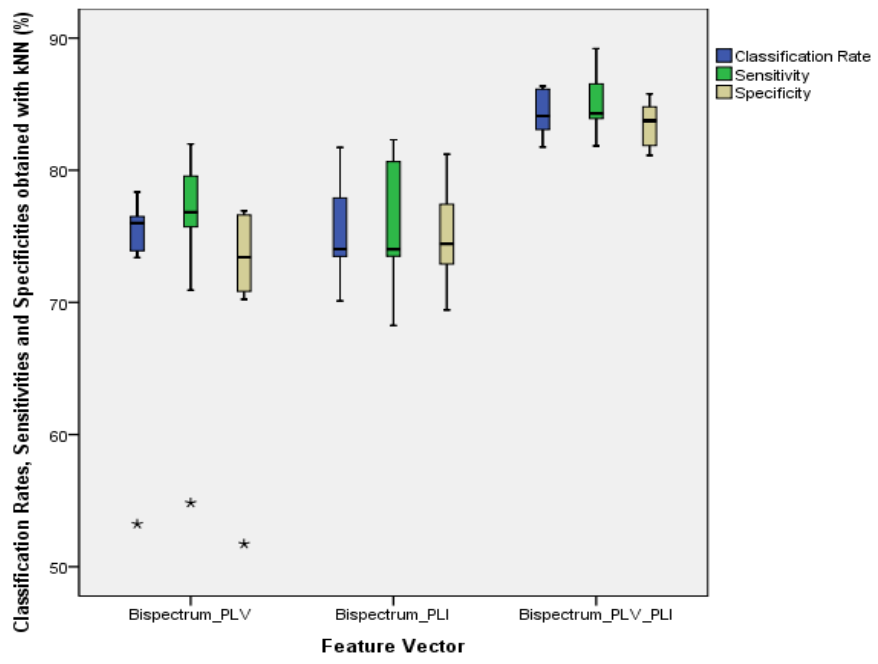

Fig. 9. The Box Plots for Classification Rate, Sensitivity and Specificity using Feature Vector Bispectrum_PLV, Bispectrum_PLI and Bispectrum_PLV_PLI - Dataset II.

The box plots for the classification rate, the sensitivity and the specificity using as feature vector Bicoherence_Bispectrum_PLV, Bicoherence_Bispectrum_PLI and Bicoherence_Bispectrum_PLV_PLI - Dataset II are presented in Fig. 10. Comparing to the other feature vectors, the smallest classification rates, sensitivities and specificities were obtained for the feature vectors from Fig. 10. 


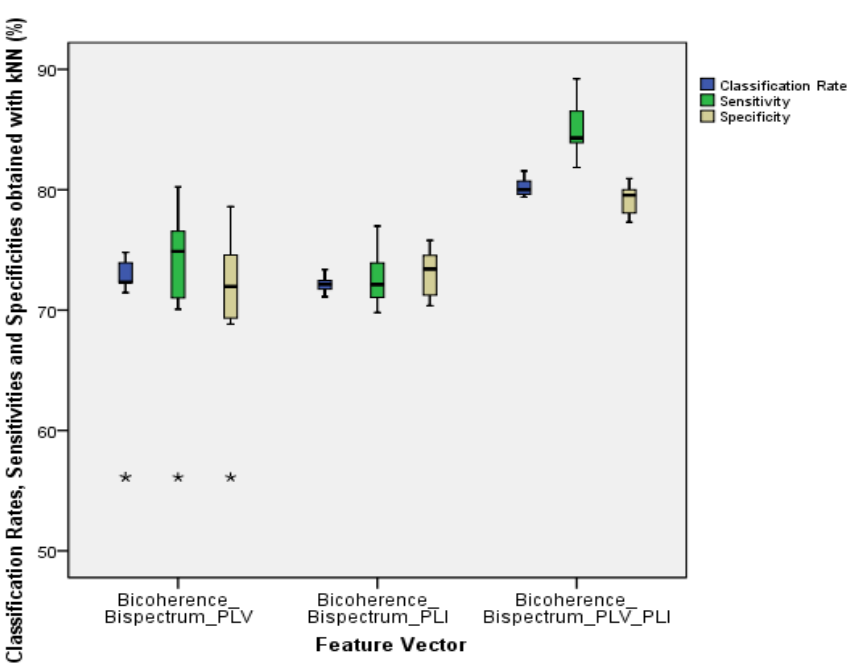

Fig. 10. The Box Plots for Classification Rate, Sensitivity and Specificity using Feature Vector Bicoherence_Bispectrum_PLV,

Bicoherence_Bispectrum_PLI and Bicoherence_Bispectrum_PLV_PLI Dataset II.

In Table II are summarized the maximum and the median of the classification rates for Dataset I and Dataset II attained with the mentioned feature vectors. The best classification rates were attained for feature vector Bicoherence_PLV_PLI for both datasets.

In [22] where a framework for achieving subject transfer strategy is proposed, the classification rates obtained for subject 9 were in the range $57.93 \%$ to $70.93 \%$ with linear discriminant analysis. The results are consistent with the results obtained with our feature vectors.

In [23] where was investigated a method based on multiple frequency spatial synthesized features with support vector machine classifier, the classification accuracies were smaller than the results reported in this paper.

TABLE II. THE MAXIMUM AND THE MEDIAN CLASSIFICATION RATES FOR THE FEATURE VECTOR USED - DATASET I AND DATASET II

\begin{tabular}{|c|c|c|c|c|}
\hline \multirow{3}{*}{ Feature Vector } & \multicolumn{3}{|l|}{ Dataset I } & Dataset II \\
\hline & \multicolumn{4}{|c|}{ Classification rate } \\
\hline & Max & Median & Max & Median \\
\hline Bicoherence_PLV & $90.62 \%$ & $86.43 \%$ & $87.28 \%$ & $84.08 \%$ \\
\hline Bicoherence_PLI & $91.74 \%$ & $86.15 \%$ & $87.45 \%$ & $84.00 \%$ \\
\hline Bicoherence_PLV_PLI & $93.90 \%$ & $89.79 \%$ & $91.86 \%$ & $88.44 \%$ \\
\hline Bispectrum_PLV & $90.31 \%$ & $82.74 \%$ & $78.35 \%$ & $76.00 \%$ \\
\hline Bispectrum_PLI & $88.45 \%$ & $82.79 \%$ & $81.73 \%$ & $74.04 \%$ \\
\hline Bispectrum_PLV_PLI & $92.76 \%$ & $88.15 \%$ & $86.37 \%$ & $84.11 \%$ \\
\hline $\begin{array}{l}\text { Bicoherence_Bispectru } \\
\text { m_PLV }\end{array}$ & $84.73 \%$ & $80.88 \%$ & $74.79 \%$ & $72.31 \%$ \\
\hline $\begin{array}{l}\text { Bicoherence_Bispectru } \\
\text { m_PLI }\end{array}$ & $85.41 \%$ & $79.98 \%$ & $76.39 \%$ & $72.28 \%$ \\
\hline $\begin{array}{l}\text { Bicoherence_Bispectru } \\
\text { m_PLV_PLI }\end{array}$ & $89.34 \%$ & $85.71 \%$ & $83.08 \%$ & $79.70 \%$ \\
\hline
\end{tabular}

In [24], [25] were manipulated only PLV and PLI without being in combinations with other methods, but more channels were taken into account. The results achieved in these two cited works are approximately the same with the results stated in this paper with feature vector Bicoherence_PLV_PLI. The maximum classification rates of $86 \%$ are attained using PLI and SVM classifier.

\section{CONCLUSION}

An offline analysis was performed on two datasets that contained 57 subjects and 9 subjects. Using combinations of bispectrum, bicoherence, phase locking value and phase lag index, nine features vectors were formed.

By means of Bicoherence_PLV_PLI as feature vector and $\mathrm{kNN}$ as classifier, the highest classification rates, sensitivities and specificities achieved for Dataset I were 93.90\%, 94.35\% and $94.29 \%$ respectively and for Dataset II were $91.86 \%$, $94.56 \%$ and $90.67 \%$, respectively.

The best classification rate attained with Bicoherence_PLV_PLI for both datasets highlights that, regardless of the database and of the conditions in which the electroencephalographic signals were recorded, the grouping of the three measures that compose this feature vector discriminates the best the two classes. The explanation might be linked to the fact that there are taken into account both the information of nonlinear interaction described by bicoherence and of the large scale synchronization described by PLV and PLI, and together they discriminate better than alone the two tasks.

The subjects from Dataset II were trained and the subjects from Dataset I were untrained. Even if the subjects from Dataset II were trained, the results obtained were worse than those for Dataset I. This fact suggests that the method used for creating feature vector Bicoherence_PLV_PLI is adequate to be used for discrimination of motor imagery tasks in a BCI, no matter the degree of subjects' training.

The future work implies testing the method on other available datasets that provide EEG signals recorded from the subjects while performing motor imagery tasks.

\section{REFERENCES}

[1] P. Arpaia, E. De Benedetto, L. Duraccio, "Design, implementation, and metrological characterization of a wearable, integrated AR-BCI handsfree system for health 4.0 monitoring", Measurement, 2021, 177:109280.

[2] A. Herweg, J. Gutzeit, S. Kleih, A. Kübler, "Wheelchair control by elderly participants in a virtual environment with a brain-computer interface (BCI) and tactile stimulation", Biological psychology, 121, 2016, pp. 117-124.

[3] A.E. Hramov, V.A. Maksimenko, A.N. Pisarchik, "Physical principles of brain-computer interfaces and their applications for rehabilitation, robotics and control of human brain states”, Physics Reports, 2021.

[4] J. Zhang, M. Wang, “A Survey on Robots Controlled by Motor Imagery Brain-Computer Interfaces", Cognitive Robotics, 2021.

[5] Janapati, R., Dalal, V., \& Sengupta, R. (2021). Advances in modern EEG-BCI signal processing: A review. Materials Today: Proceedings.

[6] M.A. Khan, R. Das, H.K. Iversen, S. Puthusserypady, "Review on motor imagery based BCI systems for upper limb post-stroke neurorehabilitation: From designing to application", Computers in Biology and Medicine, 2020, 123:103843. 
[7] R.A. Ramadan, A.V. Vasilakos, "Brain computer interface: control signals review", Neurocomputing, 223, 2017, pp. 26-44.

[8] G. Pfurtscheller, F.H. Lopes Da Silva, "Event-related EEG/MEG synchronization and desynchronization: basic principles", Clinical neurophysiology, 110(11), 1999, pp. 1842-1857.

[9] Y. Fang, M. Chen, X. Zheng, "Extracting features from phase space of EEG signals in brain-computer interfaces", Neurocomputing, 151, 2015, pp. 1477-1485.

[10] A. Kachenoura, L. Albera, L. Senhadji, P. Comon, "ICA: a potential tool for BCI system”, IEEE Signal Processing Magazine, 25(1), 2008, pp. 57-68.

[11] X. Bao, J. Hu, "Phase synchronization for classification of motor imagery EEG", Journal Of Information \&Computational Science, 5(2), 2008, pp. 949-955.

[12] https://www.gtec.at/ - accesed June 2021.

[13] J. Mellinger, G. Schalk, "BCI2000: A General-Purpose Software Platform for BCI Research", Toward Brain-Computer Interfacing, MIT Press, 2007.

[14] A. Osman, A. Robert, "Time-course of cortical activation during overt and imagined movements", Proc. Cognitive Neuroscience Annu. Meet., 1, 2001, pp.1842-1852.

[15] K.C. Chua, V. Chandran, U.R. Acharya, C.M. Lim, "Application of higher order statistics/spectra in biomedical signals-A review", Medical engineering \& physics, 32(7), 2010, pp. 679-689.

[16] S.A. Khoshnevis, R. Sankar, "Applications of higher order statistics in electroencephalography signal processing: a comprehensive survey", IEEE Reviews in biomedical engineering, 13, 2019, pp. 169-183.
[17] V. Gonuguntla, Y. Wang, K.C. Veluvolu, "Phase synchrony in subjectspecific reactive band of EEG for classification of motor imagery tasks", InEngineering in Medicine and Biology Society (EMBC), 2013, pp. 2784-2787.

[18] C.J. Stam, G. Nolte, A. Daffertshofer, "Phase lag index: assessment of functional connectivity from multi channel EEG and MEG with diminished bias from common sources", Hum. Brain Mapp., vol. 28, 2007, pp. 1178-1193.

[19] A. Swami, J.M. Mendel, C.L. Nikias, "Higher-order spectral analysis toolbox", The Mathworks Inc, 3, 1998, pp. 22-26.

[20] F. Lotte, M. Congedo, A. Le'cuyer, F. Lamarche, B. Arnaldi, "A review of classification algorithms for EEG-based brain-computer interfaces", Journal of Neural Engineering, 4(2), 2017.

[21] T.N. Alotaiby, S.A. Alshebeili, T. Alshawi, I. Ahmad, F.E. Abd ElSamie, "EEG seizure detection and prediction algorithms: a survey", EURASIP Journal on Advances in Signal Processing, 1, 2014, pp. 1-21.

[22] W. Tu, S. Sun. "A subject transfer framework for EEG classification". Neurocomputing, 82, 2012, pp. 109-116.

[23] H. Shan, H. Xu, S. Zhu, B. He, "A novel channel selection method for optimal classification in different motor imagery BCI paradigms", Biomedical engineering online, 14(1), 2015, pp. 1-18.

[24] O.D Eva, "Detection and Classification of Mu Rhythm using Phase Synchronization for a Brain Computer Interface", International Journal Of Advanced Computer Science And Applications, 7(12), 2016, pp. 321-328.

[25] O.D. Eva, A. Pasarica, D. Tarniceriu, "Phase Synchronization Based Channel Selection For A Motor Imagery Paradigm", Bul. Inst. Polit. Iasi, 63 (67):2, 2016, pp. 51-62. 